\title{
Hydroxycinnamates from elecampane (Inula helenium L.) callus culture
}

\author{
Anna Stojakowska $^{1} \cdot$ Janusz Malarz $^{1} \cdot$ Anna K. Kiss $^{2}$
}

Received: 3 June 2015/Revised: 8 January 2016/Accepted: 11 January 2016/Published online: 22 January 2016

(C) The Author(s) 2016. This article is published with open access at Springerlink.com

\begin{abstract}
Hydroalcoholic extract from Inula helenium callus tissue showed remarkable reducing capacity. An HPLC/DAD analysis revealed the presence of numerous hydroxycinnamic acid derivatives, including chlorogenic acid (5-O-CQA, $0.1 \%$ dry weight) and 3,5-dicaffeoylquinic acid (3,5-DCQA, $0.3 \%$ dry weight), which were among major constituents of the examined extract. Application of a hyphenated chromatographic methodUHPLC/DAD/MS ${ }^{\text {n }}$-allowed identification of sixteen compounds, derivatives of caffeic acid. Apart from the compounds commonly found in Inula sp., the plant material contained eight conjugates of caffeic acid with aldaric acid. The aldarates constituted over $50 \%$ of the hydroxycinnamate fraction in the examined tissue. This is the first report on the occurrence of the caffeoylaldaric acids in Inula sp. and in a plant tissue culture.
\end{abstract}

Keywords Aldaric acids - Caffeic acid derivatives . Callus culture $\cdot$ Hydroxycinnamates $\cdot$ Inula helenium

Communicated by J. V. Jorrin-Novo.

Anna Stojakowska

stoja@if-pan.krakow.pl

1 Department of Phytochemistry, Institute of Pharmacology, Polish Academy of Sciences, 12 Smętna Street, 31-343 Kraków, Poland

2 Department of Pharmacognosy and Molecular Basis of Phytotherapy, Medical University of Warsaw, 1 Banacha Street, 02-097 Warsaw, Poland

\section{Introduction}

Hydroxycinnamic acid derivatives are common plant constituents. They are present in plant tissues as free acids (i.e., coumaric, ferulic, caffeic and sinapic acids), their oligomers, and as conjugates with other compounds (most commonly with quinic acid and glucose). Mono-, di-, triand tetracaffeoylquinic acids as well as feruloylquinic and coumaroylquinic acids and conjugates of caffeic acid with tartaric acid are frequently found in Asteraceae (Jaiswal et al. 2011). Hydroxycinnamates are well-known radical scavengers and inhibitors of lipid peroxidation (Ohnishi et al. 1994; Olmos et al. 2008; Shahidi and Chandrasekara 2010). The compounds also play a role in plant defense against pathogens and insects (Kodoma et al. 1998; Leiss et al. 2009). They are of potential value in pharmacy, as antiviral, hepatoprotective and neuroprotective agents (Robinson et al. 1996; Kim et al. 2005, 2007), in cosmetics (protection against UV) and in food industry (as bioactive components of the diet and precursors for flavors). Inula helenium L., a perennial herb of the Inulae tribe (Asteraceae) is a reputed medicinal plant. The species, native of Middle Asia, widely occurs in Europe, Asia and Northern America. Underground parts of the elecampane plant, collected in the autumn after 2 or 3 years of growth, are traditionally used for medicinal purposes, for example to treat asthma, cough, bronchitis, lung disorders, tuberculosis, skin infections and helminthic diseases (Seca et al. 2014). The herbal product under the name of "Radix Inulae", "Radix Helenii" or "Rhizoma Helenii" is described in several European pharmacopeias (e.g. PF X, Ned 5, BHP). Roots of I. helenium contain up to $5 \%$ of essential oil with alantolactone, isoalantolactone (eudesmane-type sesquiterpene lactones) and monoterpenoid thymol derivatives as major constituents. Moreover, sterols, 
triterpenes and inulin (up to $44 \%$ ) were found in the roots (Blaschek et al. 1998). Literature data concerning hydroxycinnamic acid derivatives present in I. helenium are sparse. It is only recently that some reports on analysis of extracts from leaves and roots of the plant using high performance liquid chromatography with mass spectrometry (HPLC/MS) and tentative identification of phenolic compounds have been published (Jaiswal et al. 2011; Spiridon et al. 2013). Callus cultures of I. helenium maintained in our laboratory showed high total phenolic content. As far as we were aware, secondary metabolites produced by callus cultures of elecampane were not studied before. Thus, the aim of our work was to identify and quantify phenolics accumulated by the tissue.

\section{Materials and methods}

\section{Plant material}

Seeds of botanically verified I. helenium plants, of known wild origin (No. 1026/2001), were supplied by the Botanical Garden and Botanical Museum Berlin-Dahlem of the Free University in Berlin (Germany). Voucher specimen of the donor plant was deposited in the garden herbarium or in the main herbarium of the Botanical Museum. The seeds, after surface sterilization, were aseptically germinated on a half-strength, solidified (0.8\% agar) MS medium (Murashige and Skoog 1962).

\section{Callus initiation and maintenance}

Leaf explants (ca. $1 \mathrm{~cm}$ long) were excised from aseptic seedlings and subsequently inoculated onto a solidified MS medium supplemented with 2,4-D (2,4-dichlorophenoxyacetic acid, $\left.4.52 \mu \mathrm{M}^{-1}\right)$ and kinetin $\left(1.39 \mu \mathrm{M} \mathrm{l}^{-1}\right)$, containing $3 \%$ sucrose $(\mathrm{pH} 5.8$, before autoclaving). The explants were kept at $28{ }^{\circ} \mathrm{C}$, under continuous light (40 $\mu \mathrm{mol} \mathrm{m} \mathrm{m}^{-2} \mathrm{~s}^{-1}$, cool white fluorescent tubes), to initiate callus proliferation. The calli were further maintained on the culture medium of the same composition with an unaltered temperature and illumination regime. The tissue was transferred to the fresh nutrient medium every 6 weeks. The subculturing was performed by inoculating ca. $2.0 \mathrm{~g} \mathrm{FW}$ (fresh weight) of the tissue onto $50 \mathrm{ml}$ of the culture medium. For the phytochemical analysis, the calli were harvested at the end of 6 week culture period.

\section{Chemicals and solvents}

5-O-caffeoylquinic acid (chlorogenic acid, 5-O-CQA, purity $>97 \%$ by HPLC) and a standard sample of cynarin (1,3-dicaffeoylquinic acid, 1,3-DCQA, purity $>99 \%$ by
HPLC) were bought from Roth (Karlsruhe, Germany). 3,5DCQA was isolated from $L$. virosa hairy root culture (Stojakowska et al. 2012). The compound was of 90.0 \% purity (by HPLC). Folin-Ciocalteu reagent was purchased from Sigma-Aldrich Co. (St. Louis, MO, USA). Methanol $(\mathrm{MeOH})$ of analytical grade was purchased from POCh S.A. (Gliwice, Poland). Water for chromatography was purified by a Mili-Q system (Milipore Corp., Bedford, MA, USA). Acetonitrile ( $\mathrm{MeCN}$ ) and $\mathrm{MeOH}$ of gradient grade for liquid chromatography as well as glacial acetic acid and formic acid ( $\mathrm{HCOOH}$ ) of analytical grade were bought from Merck (Darmstadt, Germany).

\section{Determination of total phenolic content (TPC)}

The "total phenolic content" which reflects reducing capacity of the plant material (Huang et al. 2005) was analyzed using the Folin-Ciocalteu colorimetric method as described previously (Stojakowska et al. 2013). Results (means of three measurements) were expressed as gallic acid equivalents.

\section{Quantification of hydroxycinnamates}

The dry, pulverized plant tissue $(100 \mathrm{mg})$ was extracted twice (for $3 \mathrm{~h}$ ) with $10 \mathrm{ml}$ of $70 \% \mathrm{MeOH}$, at room temperature, on a rotary shaker (100 r.p.m.). The combined methanol extracts were evaporated in vacuo to give a dry residue which was redissolved in $1 \mathrm{ml}$ of $70 \% \mathrm{MeOH}$ and subsequently centrifuged $(11,340 \mathrm{~g}, 5 \mathrm{~min})$ just before chromatographic analysis. Analytical HPLC separations of the samples were carried out according to the method described by Malarz et al. (2013). Quantification was performed using an external standard method. The calibration curves were calculated using four concentration levels $\left(0.001,0.01,0.1\right.$ and $\left.1.0 \mathrm{mg} \mathrm{ml}^{-1}\right)$ of 5-O-CQA and 1,3-DCQA. Peak areas (at $325 \mathrm{~nm}$ ) were referred to the corresponding calibration curves. Caffeic acid derivatives were quantified as equivalents of 5-O-CQA (monocaffeoyl derivatives) and 1,3-DCQA (di- and tricaffeoyl derivatives).

\section{Identification of caffeic acid derivatives by ultra- high performance liquid chromatography with photodiode array detection and ion trap mass spectrometry (UHPLC-DAD-MS ${ }^{\mathbf{n}}$ )}

The dry, powdered tissue $(500 \mathrm{mg})$ was extracted with $10 \mathrm{ml}$ of $0.1 \% \mathrm{HCOOH} / \mathrm{MeOH}(8 / 2)$ in an ultrasonic bath $\left(40{ }^{\circ} \mathrm{C}\right)$, for $30 \mathrm{~min}$. The obtained extract was filtered through paper filter, diluted 1:2 with $0.1 \% \mathrm{HCOOH}$ and than filtered through $0.45 \mu \mathrm{m}$ Chromafil membrane (Machery-Nagel, Duren, Germany). 
$\mathrm{UHPLC} \mathrm{DAD} / \mathrm{MS}^{\mathrm{n}}$ analysis was carried out on a UHPLC-3000 RS system (Dionex, Germany) with DAD detection and an AmaZon SL ion trap mass spectrometer with ESI interface (Bruker Daltonik GmbH, Germany). The chromatographic separation was achieved on a Zorbax SB C18 column $(150 \times 2.1 \mathrm{~mm}, 1.9 \mu \mathrm{m})$ (Agilent, USA), at $25{ }^{\circ} \mathrm{C}$. The mobile phase was a mixture of $\mathrm{H}_{2} \mathrm{O} / \mathrm{MeCN} /$ $\mathrm{HCOOH}$ 95/5/0.1 (solvent A) and MeCN/HCOOH 100/0.1 (solvent B). Gradient elution conditions applied were as follows: $0-45 \mathrm{~min} 5-40 \% \mathrm{~B}$, at a flow rate of $0.2 \mathrm{ml} \mathrm{min}^{-1}$. The column was equilibrated for $8 \mathrm{~min}$ between injections. The chromatograms were assessed at $325 \mathrm{~nm}$ and UV spectra of the compounds were recorded over a range of $200-450 \mathrm{~nm}$. The column eluate was introduced directly, without splitting, into the ESI interface. The nebuliser pressure was $40 \mathrm{psi}$; dry temperature $300{ }^{\circ} \mathrm{C}$; dry gas flow $91 \mathrm{~min}^{-1}$; and capillary voltage $4.5 \mathrm{kV}$. The mass spectral analysis was performed using scan from 90 to $2200 \mathrm{~m} / \mathrm{z}$, in a negative ion mode. The $\mathrm{MS}^{2}$ fragmentation spectra were acquired for the most abundant ions at a given time.

\section{Results and discussion}

After 6 weeks of culture, FW and DW (dry weight) of $I$. helenium calli reached $8.86 \pm 1.60$ and $0.28 \pm 0.06 \mathrm{~g}$ per culture vessel, respectively. Longer cultivation led to the growth arrest and a change in tissue color from green to dark brown. TPC in the 6-week-old callus tissue, calculated on a DW basis, reached $6.78 \pm 0.37 \%\left(67.8 \mathrm{mg} \mathrm{g}^{-1}\right)$, expressed as gallic acid equivalents. According to literature data (Kähkönen et al. 1999; Malarz et al. 2013), reducing capacities of Cichorium intybus leaves and Aronia melanocarpa fruits which are known sources of antioxidants were lower than that estimated for the examined tissue. Callus cultures of Inula crithmoides, grown on MS medium supplemented with 2,4-D $\left(1.0 \mathrm{mg} \mathrm{l}^{-1}\right)$, contained 4.4-28.3 $\mathrm{mg} \mathrm{g}^{-1}$ of phenolics. The content depended on the phase of growth (maximum at the stationary phase) and was lower in cultures grown in the dark (Bucchini et al. 2013). TPC estimated in I. helenium roots ranged from 3.5 to $5.8 \mathrm{mg} \mathrm{g}^{-1}$ (Wang et al. 2013).

Preliminary HPLC/DAD analysis of hydroalcoholic extracts from I. helenium calli revealed the presence of numerous compounds with absorption maxima at about $325 \mathrm{~nm}$, which corresponded to those of hydroxycinnamic acid derivatives. Three of the compounds (5-O-CQA, 1,3DCQA and 3,5-DCQA) were identified based on their UV spectra, retention times and co-chromatography with standards. To identify the remaining constituents of the extract, HPLC/DAD/MS ${ }^{\mathrm{n}}$ technique was applied. Based on $\mathrm{UV}-\mathrm{V}$ is spectra and $\mathrm{MS}^{\mathrm{n}}$, we were able to identify 16 compounds (Fig. 1). In accordance with Clifford et al. (2005), the negative ion $\mathrm{MS}^{2}$ and $\mathrm{MS}^{3}$ fragmentation patterns of compounds 1-3 allowed their identification as 3-, 5-, and 4-O-CQA (IUPAC numbering system), while compounds 5, 9-11 and $\mathbf{1 3}$ were assigned as isomers of DCQA (Table 1). Peaks 4, 6-8, 12 and 14-16 showed a cleavage of two to four caffeoyl [M $-\mathrm{H}-162]^{-}$moieties resulting in $\mathrm{m} / \mathrm{z} 209$ fragment (Table 1), indicating the presence of glucaric acid or galactaric acid, the so-called aldaric acids (Ruiz et al. 2013; Lorentz et al. 2012). However, up to now, only the presence of hydroxycinnamic acid glucarates has been reported (Maas et al. 2009; Lorentz et al. 2012; Ruiz et al. 2014). The presence of this rare type of compounds was not reported in Inula sp. before, although the presence of di- tri- and tetracaffeoylglucaric acid derivatives was demonstrated in other genera of Asteroideae subfamily such as Leontopodium, Gnaphalium and Eupatorium (Schwaiger et al. 2005; Maas et al. 2009; Cicek et al. 2012). Jaiswal et al. (2011) identified six hydroxycinnamic acid derivatives in the extract from aerial parts of $I$. helenium, including three monocaffeoylquinic acids (1-O-, 3-O-, and 5-O-CQA), 5- $O$-feruloylquinic acid, 5-O-p-coumaroylquinic acid, and 3-Ocaffeoyl-4- $O$ - $p$-coumaroylquinic acid. According to Spiridon et al. (2013), roots of the plant contained: caffeic acid, caffeic acid hexose of undetermined structure, 5-O-CQA, 5-O-feruloylquinic acid, ferulic acid-4- $O$-glucoside, DCQA (structure not determined), 3- $O$-feruloyl-4- $O$-caffeoylquinic acid, and 3,4,5-tri- $O$-caffeoylquinic acid. Our research revealed the presence of 16 caffeic acid derivatives in the examined tissue, whereas derivatives of ferulic and $p$-coumaric acids were absent from the analyzed calli.

Quantification of caffeic acid derivatives in I. helenium callus culture was done by HPLC/PDA (results are shown in Table 1). Total content of hydroxycinnamic acid derivatives in the analyzed plant material, calculated as a sum of detected compounds, was about $1.4 \%$ (on a DW basis). Total content of caffeoylquinates (ca. $0.7 \%$ ) was slightly lower than that of caffeoyl aldarates (ca. $0.8 \%$ ). Five major compounds, i.e., 5-O-CQA, 3,5-DCQA, 1,5DCQA, and tricaffeoyl aldarates (I) and (II) constituted over $70 \%$ of the hydroxycinnamate fraction. In vitro cultures of Asteraceae are good producers of hydroxycinnamates (mainly caffeoylquinates and caffeoyltartrates). The highest total content of the compounds (up to $7 \% \mathrm{DW}$ ) was found in a hairy root culture of Cichorium intybus L. (Malarz et al. 2013). Hairy roots of Echinacea purpurea (L.) Moench. (Abbasi et al. 2007), Lactuca virosa L. (Stojakowska et al. 2012) and Rhaponticum carthamoides (Willd.) Iljin (Skała et al. 2015) also accumulated substantial amounts of the hydroxycinnamic acid derivatives. Caffeic acid derivative contents in callus cultures of Asteraceae were lower than those in the corresponding root 


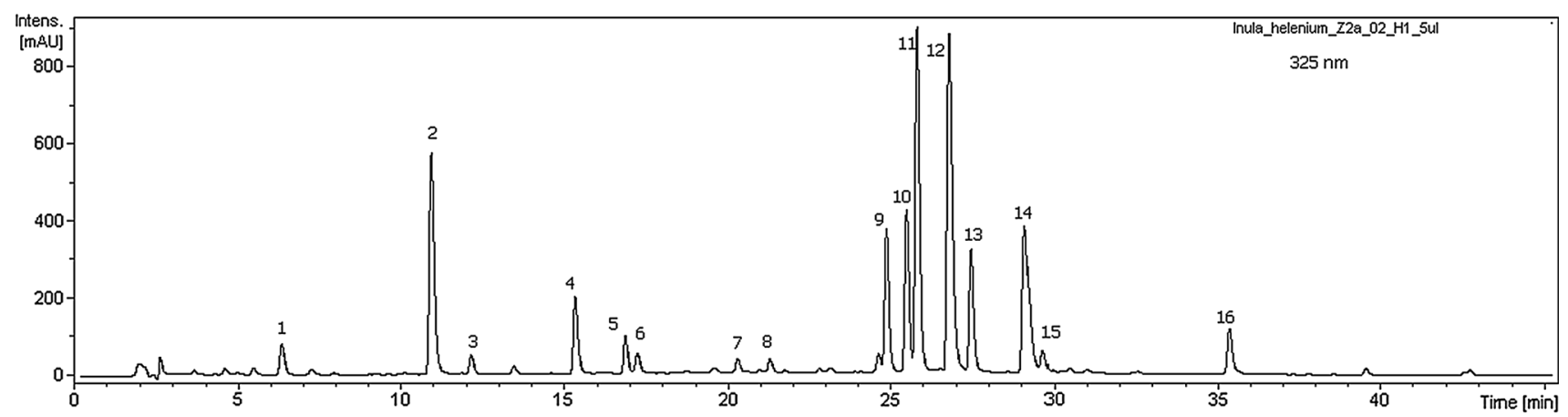

Fig. 1 HPLC-UV chromatogram of I. helenium extract at a concentration of $25 \mathrm{mg} / \mathrm{ml}$ (injection volume-5 $\mu \mathrm{l}$ ), acquired at $325 \mathrm{~nm}$

Table 1 Retention times, UV maxima, MS ${ }^{\mathrm{n}}$ data in the negative ion mode, and contents of caffeic acid derivatives detected in Inula helenium callus extract

\begin{tabular}{lllllll}
\hline No & Compound & Rt $(\mathrm{min})$ & $\mathrm{UV}(\mathrm{nm})$ & {$[\mathrm{M}-\mathrm{H}]^{-}$} & Product ions main peaks $^{\mathrm{a}}$ & Content $^{\mathrm{b}}(\% \mathrm{DW})$ \\
\hline 1 & 3- $O$-caffeoylquinic acid & 6.3 & 216,324 & 353 & $\mathbf{1 9 1}, 179$ & $0.014 \pm 0.0005$ \\
2 & 5-O-caffeoylquinic acid & 10.9 & 216,326 & 353 & $\mathbf{1 9 1}$ & $0.101 \pm 0.0019$ \\
3 & 4-O-caffeoylquinic acid & 12.1 & 213,325 & 353 & $191,179, \mathbf{1 7 3}$ & $0.010 \pm 0.0010$ \\
4 & Dicaffeoyl aldarate (I) & 15.3 & 215,324 & 533 & $\mathbf{3 7 1}, 209$ & $0.052 \pm 0.0025$ \\
5 & 1,3-Di- $O$-caffeoylquinic acid & 16.8 & 215,325 & 515 & $\mathbf{3 5 3}, 335,191,179$ & $0.018 \pm 0.0002$ \\
6 & Dicaffeoyl aldarate (II) & 17.3 & 212,326 & 533 & $\mathbf{3 7 1}, 209$ & $0.013 \pm 0.0020$ \\
7 & Dicaffeoyl aldarate (III) & 20.4 & 214,326 & 533 & $\mathbf{3 7 1}, 209$ & $0.010 \pm 0.0001$ \\
8 & Dicaffeoyl aldarate (IV) & 21.3 & 214,326 & 533 & $\mathbf{3 7 1}, 209$ & $0.010 \pm 0.0005$ \\
9 & 3,4-Di- $O$-caffeoylquinic acid & 24.9 & 216,326 & 515 & $\mathbf{3 5 3}, 335,299,255,203,191,179,173$ & $0.070 \pm 0.0003$ \\
10 & 1,5-Di- $O$-caffeoylquinic acid & 25.4 & 215,328 & 515 & $\mathbf{3 5 3}, 335,191$ & $0.107 \pm 0.0049$ \\
11 & 3,5-Di- $O$-caffeoylquinic acid & 25.8 & 215,327 & 515 & $\mathbf{3 5 3}, 191$ & $0.259 \pm 0.0088$ \\
12 & Tricaffeoyl aldarate (I) & 26.7 & 217,327 & 695 & $\mathbf{5 3 3}, \mathbf{3 7 1}, 209$ & $0.400 \pm 0.0022$ \\
13 & 4,5-Di- $O$-caffeoylquinic acid & 27.4 & 215,327 & 515 & $\mathbf{3 5 3}, 317,299,255,203,173$ & $0.075 \pm 0.0030$ \\
14 & Tricaffeoyl aldarate (II) & 29.1 & 216,328 & 695 & $\mathbf{5 3 3}, \mathbf{3 7 1}, 209$ & $0.164 \pm 0.0008$ \\
15 & Tricaffeoyl aldarate (III) & 29.7 & 216,327 & 695 & $\mathbf{5 3 3}, \mathbf{3 7 1}, 209$ & $0.044 \pm 0.0008$ \\
16 & Tetracaffeoyl aldarate & 35.3 & 217,328 & 857 & $\mathbf{6 9 8}, 533,371,209$ & $0.077 \pm 0.0051$ \\
\hline
\end{tabular}

${ }^{\mathrm{a}} \mathrm{MS}^{2}$ ions in bold-most abundant ion peak

${ }^{\mathrm{b}}$ Data are means of three measurements \pm SD

cultures. Calli of $C$. intybus and L. virosa accumulated $1.4 \%$ DW and $0.7 \%$ DW of caffeic acid derivatives, respectively (Stojakowska et al. 2012; Malarz et al. 2013). The present study is, to our knowledge, the first report on the occurrence of caffeoyl aldarates in in vitro-cultured plant tissues.

Author contribution statement Anna K. Kiss performed UHPLC/DAD/MS ${ }^{\mathrm{n}}$ analysis and contributed in writing of the manuscript. Janusz Malarz and Anna Stojakowska have contributed equally to the remaining part of this publication.

Acknowledgments The financial support of the Ministry of Science and Higher Education, Poland (statutory activity funding), is gratefully acknowledged.

\section{Compliance with ethical standards}

Conflict of interest The authors declare that there is no conflict of interest.

Open Access This article is distributed under the terms of the Creative Commons Attribution 4.0 International License (http://creative commons.org/licenses/by/4.0/), which permits unrestricted use, distribution, and reproduction in any medium, provided you give appropriate credit to the original author(s) and the source, provide a link to the Creative Commons license, and indicate if changes were made.

\section{References}

Abbasi BH, Tian C-L, Murch SJ, Saxena PK, Liu C-Z (2007) Lightenhanced caffeic acid derivatives biosynthesis in hairy root cultures of Echinacea purpurea. Plant Cell Rep 26:1367-1372 
Blaschek W, Hänsel R, Keller K, Reichling J, Rimpler H, Schneider G (1998) Hagers Handbuch der Pharmazeutischen Praxis, vol 2: Drogen A-K. Springer Verlag, Berlin-Heidelberg, pp 526-530

Bucchini A, Giamperi L, Ricci D (2013) Total polyphenol content, in vitro antifungal and antioxidant activities of callus cultures from Inula crithmoides. Nat Prod Commun 8:1587-1590

Cicek SS, Untersulzner C, Schwaiger S, Zidorn C (2012) Caffeoyl-Dglucaric acid derivatives in the genus Gnaphalium (Asteraceae: Gnaphalieae). Rec Nat Prod 6:311-315

Clifford MN, Knight S, Kuhnert N (2005) Discriminating between the six isomers of dicaffeoylquinic acid by LC-MS ${ }^{\mathrm{n}}$. J Agric Food Chem 53:3821-3832

Huang D, Ou B, Prior RL (2005) The chemistry behind antioxidant capacity assays. J Agric Food Chem 53:1841-1856

Jaiswal R, Kiprotich J, Kuhnert N (2011) Determination of the hydroxycinnamate profile of 12 members of the Asteraceae family. Phytochemistry 72:781-790

Kähkönen MP, Hopia AI, Vuorela HJ, Rauha J-P, Pihlaja K, Kujala TS, Heinonen M (1999) Antioxidant activity of plant extracts containing phenolic compounds. J Agric Food Chem 47:3954-3962

Kim S-S, Park R-Y, Jeon H-J, Kwon Y-S, Chun W (2005) Neuroprotective effects of 3,5-dicaffeoylquinic acid on hydrogen peroxide-induced cell death in SH-SY5Y cells. Phytother Res 19:243-245

Kim KH, Kim YH, Lee KR (2007) Isolation of quinic acid derivatives and flavonoids from the aerial parts of Lactuca indica L. and their hepatoprotective activity in vitro. Bioorg Med Chem Lett 17:6739-6743

Kodoma M, Wada H, Otani H, Kohmoto K, Kimura Y (1998) 3,5-Di$O$-caffeoylquinic acid, an infection-inhibiting factor from Pyrus pyrifolia induced by infection with Alternaria alternata. Phytochemistry 47:371-373

Leiss KA, Maltese F, Choi YH, Verpoorte R, Klinkhamer PGL (2009) Identification of chlorogenic acid as a resistance factor for thrips in Chrysanthemum. Plant Physiol 150:1567-1575

Lorentz P, Conrad J, Bertrams J, Berger M, Duckstein S, Meyer U, Stintzing FC (2012) Investigations into the phenolic constituents of Dog's mercury (Mercurialis perennis L.) by LC-MS/MS and GC-MS analyses. Phytochem Anal 23:60-71

Maas M, Petereit F, Hensel A (2009) Caffeic acid derivatives from Eupatorium perfoliatum L. Molecules 14:6-45

Malarz J, Stojakowska A, Kisiel W (2013) Long-term cultured hairy roots of chicory: a rich source of hydroxycinnamates and 8-deoxylactucin glucoside. Appl Biochem Biotechnol 171: 1589-1601

Murashige T, Skoog F (1962) A revised medium for rapid growth and bioassays with tobacco tissue cultures. Physiol Plant 15:473-497
Ohnishi M, Morishita H, Iwahashi H, Toda S, Shirataki Y, Kimura M, Kido R (1994) Inhibitory effects of chlorogenic acids on linoleic acid peroxidation and haemolysis. Phytochemistry 36:579-583

Olmos A, Giner RM, Recio MC, Ríos JL, Gil-Benso R, Máñez S (2008) Interaction of dicaffeoylquinic derivatives with peroxynitrite and other reactive nitrogen species. Arch Biochem Biophys 475:66-71

Robinson WE Jr, Reinecke MG, Abdel-Malek S, Jia Q, Chow SA (1996) Inhibitors of HIV-1 replication that inhibit HIV integrase. Proc Natl Acad Sci 93:6326-6331

Ruiz A, Mardones C, Vergara C, Hermosín-Gutiérrez I, von Baer D, Hinrichsen P, Rodrigez R, Arribillaga D, Dominguez E (2013) Analysis of hydroxycinnamic acids derivatives in calafate (Berberis microphylla G. Forst) berries by liquid chromatography with photodiode array and mass spectrometry detection. J Chromatogr A 1281:38-45

Ruiz A, Mardones C, Vergara C, von Baer D, Hinrichsen P, GómezAlonso S, Gómez MV, Hermosín-Gutiérrez I (2014) Isolation and structural elucidation of anthocyanidin 3,7- $\beta$ - $O$-diglucosides and caffeoylglucaric acids from calafate berries. J Agric Food Chem 62:6918-6925

Schwaiger S, Cervellati R, Seger C, Ellmerer E, About N, Renimel I, Godenir C, André P, Gafner F, Stuppner H (2005) Leontopodic acid: a novel highly substituted glucaric acid derivative from Edelweiss (Leontopodium alpinum Cass.) and its antioxidative and DNA protecting properties. Tetrahedron 61:4621-4630

Seca AML, Grigore A, Pinto DCGA, Silva AMS (2014) The genus Inula and their metabolites: from ethnopharmacological to medicinal uses. J Ethnopharmacol 154:286-310

Shahidi F, Chandrasekara A (2010) Hydroxycinnamates and their in vitro and in vivo antioxidant activities. Phytochem Rev 9:147-170

Skała E, Kicel, Olszewska MA, Kiss AK, Wysokińska H (2015) Establishment of hairy root cultures of Rhaponticum carthamoides (Willd.) Iljin for the production of biomass and caffeic acid derivatives. BioMed Res International 2015:181098

Spiridon I, Nechita CB, Niculaua M, Silion M, Armatu A, Teacă C-A, Bodîrlǎu R (2013) Antioxidant and chemical properties of Inula helenium root extracts. Cent Eur J Chem 11:1699-1709

Stojakowska A, Malarz J, Szewczyk A, Kisiel W (2012) Caffeic acid derivatives from a hairy root culture of Lactuca virosa. Acta Physiol Plant 34:291-298

Stojakowska A, Michalska K, Malarz J, Beharav A, Kisiel W (2013) Root tubers of Lactuca tuberosa as a source of antioxidant phenolic compounds and new furofuran lignans. Food Chem 138:1250-1255

Wang J, Zhao Y-M, Tian Y-T, Yan C-L, Guo C-Y (2013) Ultrasoundassisted extraction of total phenolic compounds from Inula helenium. Sci World J 2013:157527 